\title{
DUALITIES IN
}

\section{GAUGE AND}

\section{STRING}

\section{THEORIES}


This page is intentionally left blank 
Proceedings of the APCTP Winter School

\section{DUALITIES IN}

\section{GAUGE AND}

\section{STRING}

\section{THEORIES}

Sorak Mountain Resort, Korea

17-28 February 1997

Edited by

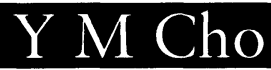

APCTP, Korea

\section{S Nam}

Kyung Hee University, Korea 


\title{
Published by
}

World Scientific Publishing Co. Pte. Ltd.

P O Box 128, Farrer Road, Singapore 912805

USA office: Suite 1B, 1060 Main Street, River Edge, NJ 07661

UK office: 57 Shelton Street, Covent Garden, London WC2H 9HE

\author{
Library of Congress Cataloging-in-Publication Data \\ APCTP Winter School (1997 : Sorak Mountain Resort, Korea) \\ Dualities in gauge and string theories : proceedings \\ of APCTP Winter School, Sorak Mountain Resort, Korea, \\ 17-28 February 1997 / edited by Y. M. Cho, S. Nam. \\ p. cm. \\ ISBN 9810235860 (alk. paper) \\ 1. Gauge fields (Physics) -- Congresses. 2. String \\ models -- Congresses. 3. Duality (Nuclear physics) - \\ - Congresses. I. Cho, Y. M. II. Nam, S. III. Title. \\ QC793.3.F5A63 1997 \\ 530.14'35--dc21 \\ 98-8803 \\ CIP
}

British Library Cataloguing-in-Publication Data

A catalogue record for this book is available from the British Library.

Copyright ( 1998 by World Scientific Publishing Co. Pte. Ltd.

All rights reserved. This book, or parts thereof, may not be reproduced in any form or by any means, electronic or mechanical, including photocopying, recording or any information storage and retrieval system now known or to be invented, without written permission from the Publisher.

For photocopying of material in this volume, please pay a copying fee through the Copyright Clearance Center, Inc., 222 Rosewood Drive, Danvers, MA 01923, USA. In this case permission to photocopy is not required from the publisher.

Printed in Singapore by Uto-Print 


\section{Preface}

There has been a very impressive progress in the area of gauge and string theories, through the use of the idea of duality. This made some of nonperturbative analysis of gauge theories possible and made string theory come back in full force an in a richer form as M-theory. Through the establishment of the Asia-Pacific Center for Theoretical Physics (APCTP) in Seoul, Korea, in June 1996, it has now become possible to bring some of the excitements in the field to the Asia Pacific region without any delay.

The purpose of the school was to provide a basic framework for the study of the dualities in gauge and string theories, for graduate students and postdocs as well as to those interested in the field. The school ran for two weeks. The first week, took place at the APCTP building in Seoul, had the introductory lectures and seminars and had the character of preparation for the second week. The second week's program was held at the Sorak Park Hotel located at Sokcho (east coast of Korea), and had the lectures by invited speakers as well as seminars. More than 100 physicists from all over the world have participated to make this winter school very successful. We thank them all.

The school was organized by the Asia-Pacific Center for Theoretical Physics, and partially supported by the Daewoo Foundation and Center for Theoretical Physics at Seoul National University. We thank them for the kind support.

Seoul

March, 1997
Y. M. Cho

Asia-Pacific Center for Theoretical Physics

S. Nam

Kyung Hee University 


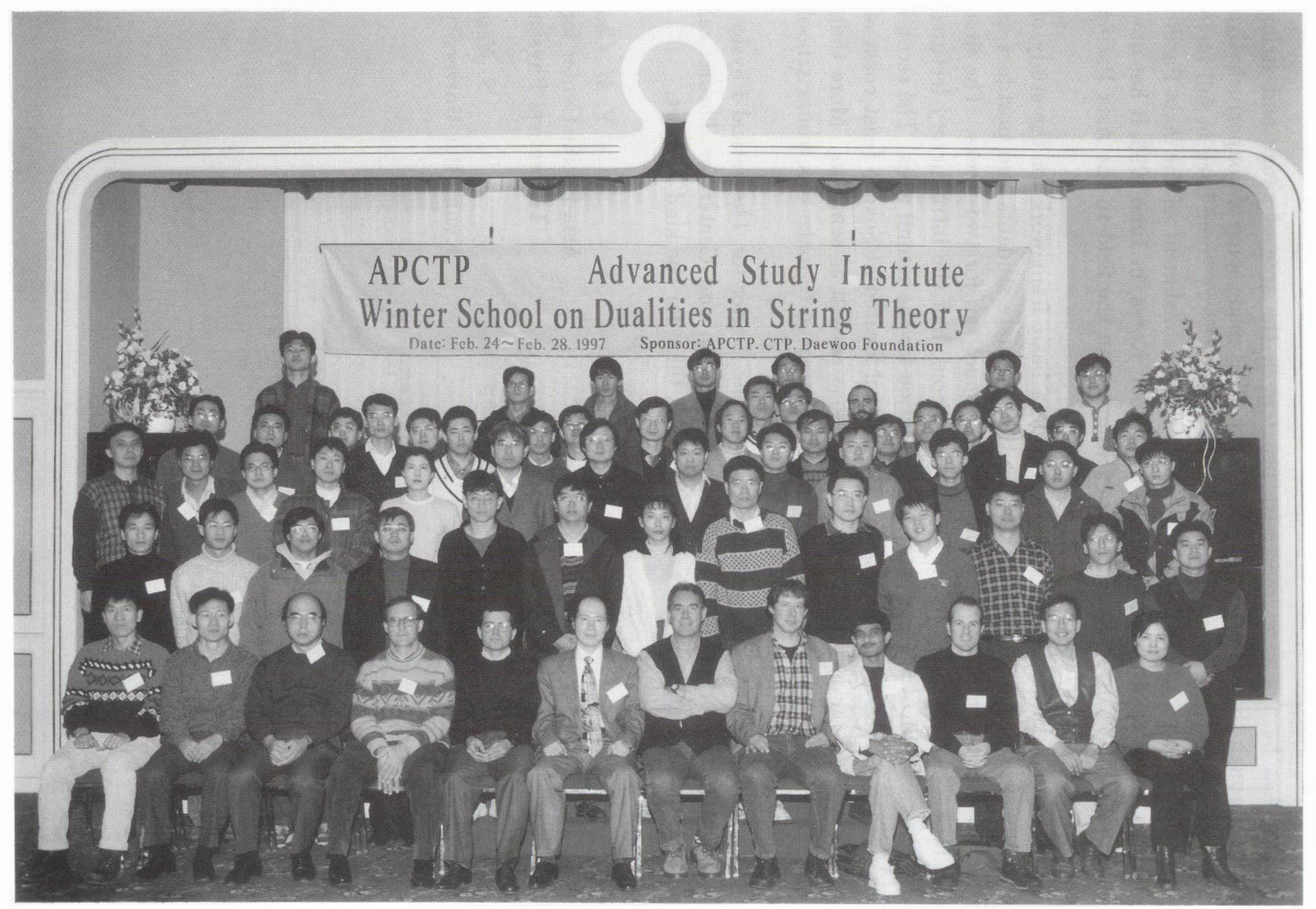




\section{Contents}

Preface

V

\section{Lectures}

Supermembranes: An Introduction M. J. Duff

An Introduction to $p$-branes

K. S. Stelle

Notes on Matrix Strings and Fivebranes

R. Dijkgraaf, E. Verlinde and H. Verlinde*

Intersecting Branes

BPS Bound States, Supermembranes, and T-Duality in M Theory

J. G. Russo

$D=6, N=1$ String Vacua and Duality

L. E. Ibáñez and A. M. Uranga

Flat Symplectic Bundles of $N$-Extended Supergravities,

Central Charges and Black-Hole Entropy

L. Andrianopoli, R. D'Auria and S. Ferrara*

Black Hole Thermodynamics and String Theory

S. R. Das

\section{Seminars}

One-Instanton Calculations in $N=2$ Supersymmetric Gauge Theories K. Ito

Field Theory on Coadjoint Orbit and Self-Dual Chern-Simons Solitons P. Oh

Cohomological Yang-Mills Theory in Eight Dimensions

L. Baulieu, H. Kanno* and I. M. Singer 
Charged BTZ Black Hole as a Global Vortex in Anti-de Sitter Spacetime:

A Bridge by Duality

N. Kim, Y. Kim* and K. Kimm

Tensionless Gravitational String in $D=6, N=1$ Heterotic String Vacua

N. Kim* ${ }^{*}$ S. J. Rey, S. Theisen and S. Yankielowicz

Quantum Ergoregion Instability

G. Kang 